\title{
On the k-Lucas Numbers of Arithmetic Indexes
}

\author{
Sergio Falcon \\ Department of Mathematics and Institute for Applied Microelectronics (IUMA), \\ University of Las Palmas de Gran Canaria, Las Palmas, Spain \\ Email: sfalcon@dma.ulpgc.es
}

Received January 23, 2012; revised September 5, 2012; accepted June 12, 2012

\begin{abstract}
In this paper, we study the k-Lucas numbers of arithmetic indexes of the form an $+r$, where $n$ is a natural number and $r$ is less than $r$. We prove a formula for the sum of these numbers and particularly the sums of the first k-Lucas numbers, and then for the even and the odd k-Lucas numbers. Later, we find the generating function of these numbers. Below we prove these same formulas for the alternated k-Lucas numbers. Then, we prove a relation between the k-Fibonacci numbers of indexes of the form $2^{r} n$ and the k-Lucas numbers of indexes multiple of 4 . Finally, we find a formula for the sum of the square of the k-Fibonacci even numbers by mean of the k-Lucas numbers.
\end{abstract}

Keywords: k-Fibonacci Numbers; k-Lucas Numbers; Generating Function

\section{Introduction}

Let us remember the k-Lucas numbers $L_{k, n}$ are defined [1] by the recurrence relation $L_{k, n+1}=k L_{k, n}+L_{k, n-1}$ with the initial conditions $L_{k, 0}=2, L_{k, 1}=k$

Among other properties, the Binnet Identity establishes $L_{k,}=\sigma_{1}^{n}+\sigma_{2}^{n}$ being $\sigma_{1}=\frac{k+\sqrt{k^{2}+4}}{2}$ and $\sigma_{2}=\frac{k-\sqrt{k^{2}+4}}{2}$ the characteristic roots of the recurrence equation $r^{2}-k r-1=0$.

Evidently, $\sigma_{1} \sigma_{2}=-1, \sigma_{1}+\sigma_{2}=k, \sigma_{1}-\sigma_{2}=\sqrt{k^{2}+4}$.

Moreover, it is verified [1, Theorem 2.4] that $L_{k, n}=F_{k, n-1}+F_{k, n+1}$.

If we apply iteratively the equation $L_{k, n+1}=k L_{k, n}+L_{k, n-1}$ then we will find a formula that relates the $\mathrm{k}$-Lucas numbers to the $\mathrm{k}$-Fibonacci numbers:

$$
L_{k, n}=L_{k, n-(p-1)} F_{k, p}+L_{k, n-p} F_{k, p-1}
$$

This formula is similar to the Convolution formula for the k-Fibonacci numbers $F_{k, n+m}=F_{k, n+1} F_{k, m}+F_{k, n} F_{k, m-1}$ $[2,3]$.

Moreover, we define $L_{k,-n}=(-1)^{n+1} L_{k, n}$. Then, if we do $\mathrm{p}=-\mathrm{n}$ in Formula (1.1) obtain $L_{k, n}=(-1)^{n+1}\left(L_{k, 2 n+1} F_{k, n}-L_{k, 2 n} F_{k, n+1}\right)$.

\section{On the k-Lucas Numbers of Arithmetic Index}

We begin this section with a formula that relates each other some k-Lucas numbers.

\subsection{Theorem 1 (The k-Lucas Numbers of Arithmetic Index)}

If a is a nonnull natural number and $r=0,1,2, \cdots a-1$, then

$$
L_{k, a(n+1)+r}=L_{k, a} L_{k, a n+r}-(-1)^{a} L_{k, a(n-1)+r}
$$

Proof. In [4] it is proved

$$
\begin{aligned}
F_{k, a(n+1)+r} & =\left(F_{k, a-1}+F_{k, a+1}\right) F_{k, a n+r}-(-1)^{a} F_{k, a(n-1)+r} \text {. Then } \\
& L_{k, a(n+1)+r}=F_{k, a(n+1)+r-1}+F_{k, a(n+1)+r+1} \\
& =\left(F_{k, a-1}+F_{k, a+1}\right) F_{k, a n+r-1} \\
& -(-1)^{a} F_{k, a(n-1)+r-1}+\left(F_{k, a-1}+F_{k, a+1}\right) F_{k, a n+r+1} \\
& -(-1)^{a} F_{k, a(n-1)+r+1} \\
& =L_{k, a} L_{k, a n+r}-(-1)^{a} L_{k, a(n-1)+r}
\end{aligned}
$$

If $r=0$, then $L_{k, a(n+1)}=L_{k, a} L_{k, a n}-(-1)^{a} L_{k, a(n-1)}$

In this case, if $a=2 p+1$, then an odd k-Lucas number can be expressed in the form

$$
L_{k,(2 p+1)(n+1)}=L_{k, 2 p+1} L_{k,(2 p+1) n}+L_{k,(2 p+1)(n-1)}
$$

Applying iteratively Formula (2.1), the general term, for $0 \leq m \leq n$, can be written like a non-linear combination of the form $L_{k, a n+r}=\sum_{j=0}^{m}(-1)^{j(a+1)}\left(\begin{array}{c}m \\ j\end{array}\right) L_{k, a}^{m-j} L_{k, a(n-m-j)+r}$

In particular, if $m=n$, then

$$
L_{k, a n+r}=\sum_{j=0}^{n}(-1)^{j(a+1)}\left(\begin{array}{l}
n \\
j
\end{array}\right) L_{k, a}^{n-j} L_{k, r-a j}
$$




\subsection{Generating Function of the Sequence $\left\{\boldsymbol{L}_{\boldsymbol{k}, \boldsymbol{a n}+r}\right\}$}

Let $l(k, x ; a, r)$ be the generating function of the sequence $\left\{L_{k, a n+r}\right\}$. That is,

$$
\begin{aligned}
& l(k, x ; a, r)=L_{k, r}+L_{k, a+r} X \\
& +L_{k, a+2 r} x^{2}+L_{k, a+3 r} x^{3}+\cdots
\end{aligned}
$$

Then,

$$
\begin{aligned}
& L_{k, a} x \cdot l(k, x ; a, r)=L_{k, a} L_{k, r} x+L_{k, a} L_{k, a+r} x^{2} \\
& +L_{k, a} L_{k, a+2 r} x^{3}+\cdots
\end{aligned}
$$

and

$$
\begin{aligned}
& (-1)^{a} x^{2} \mathfrak{1}\left(\begin{array}{llll}
k & \times & a & r
\end{array}\right) \\
& =(-1)^{a} L_{k, r} x^{2}+(-1)^{a} L_{k, a+r} x^{3}+\cdots
\end{aligned}
$$

from where

$$
l(k, x ; a, r) \cdot\left(1-L_{k, a} x+(-1)^{a} x^{2}\right)=L_{k, r}+\left(L_{k, a+r}-L_{k, a}\right) x
$$

no more to take into account Formula (2.1). So, the generating function of the sequence $\left\{L_{k, a n+r}\right\}$ is

$$
l(k, x ; a, r)=\frac{\left(L_{k, r}+L_{k, a+r}-L_{k, a} L_{k, r}\right) x}{1-L_{k, a} x+(-1)^{a} x^{2}} .
$$

As particular case, if $a=1$, then $r=0$ and the generating function of the k-Lucas sequence $\left\{L_{k, n}\right\}$ is

$I(k, x)=\frac{2-k x}{1-k x-x^{2}}$, that, for the classical Lucas sequence is $l(x)=\frac{2-x}{1-x-x^{2}}$

If we want to take out the two bisection sequences of the classical Lucas sequence $(k=1)$, the respective generating functions are $a=2$ and $r=0$;

$l(x ; 2,0)=\frac{2-3 x}{1-3 x+x^{2}}$ that generates the sequence

$$
\left\{L_{2 n}\right\}=\{2,3,7,18,47,123, \cdots\}
$$

$a=2$ and $r=1: \quad l(x ; 2,1)=\frac{1+x}{1-3 x+x^{2}}$ that generates the sequence $\left\{L_{2 n+1}\right\}=\{1,4,11,29,76,199, \cdots\}$.

\subsection{Theorem 2 (Sum of the k-Lucas Numbers of Arithmetic Index)}

If $\mathrm{a}$ is a nonnull natural number and $r=0,1,2, \cdots a-1$, then

$$
\sum_{j=0}^{n} L_{k, a j+r}=\frac{L_{k, a(n+1)+r}-(-1)^{a} L_{k, a n+r}+(-1)^{r} L_{k, a-r}-L_{k, r}}{L_{k, a}-(-1)^{a}-1}
$$

Proof.

$$
\begin{aligned}
\sum_{j=0}^{n} L_{k, a j+r} & =\sum_{j=0}^{n}\left(\sigma_{1}^{a j+r}+\sigma_{2}^{a j+r}\right)=\frac{\sigma_{1}^{a n+r}-\sigma_{1}^{r}}{\sigma_{1}^{a}-1}+\frac{\sigma_{2}^{a n+r}-\sigma_{2}^{r}}{\sigma_{2}^{a}-1} \\
& =\frac{(-1)^{a} \sigma_{1}^{a n+r}-\sigma_{1}^{a(n+1)+r}-\sigma_{1}^{r} \sigma_{2}^{a}+\sigma_{1}^{r}+(-1)^{a} \sigma_{2}^{a n+r}-\sigma_{2}^{a(n+1)+r}-\sigma_{2}^{r} \sigma_{1}^{a}+\sigma_{1}^{r}}{(-1)^{a}-\sigma_{1}^{a}-\sigma_{2}^{a}+1} \\
& =\frac{(-1)^{a} L_{k, a n+r}-L_{k, a(n+1)+r}-(-1)^{r} L_{k, a-r}+L_{k, r}}{-L_{k, a}+(-1)^{a}+1}
\end{aligned}
$$

because

$$
\sigma_{1}^{r} \sigma_{2}^{a}+\sigma_{1}^{a} \sigma_{2}^{r}=\sigma_{1}^{r} \sigma_{2}^{a-r} \sigma_{2}^{r}+\sigma_{1}^{a-r} \sigma_{1}^{r} \sigma_{2}^{r}=(-1)^{r}\left(\sigma_{2}^{a-r}+\sigma_{1}^{a-r}\right)
$$

and after applying the formula for the sum of a geometric progression.

\subsection{Corollary 1 (Sum of Consecutive Odd k-Lucas Numbers)}

If $r=0$ and $a=2 p+1$, Equation (2.2) is

$$
\sum_{j=0}^{n} L_{k,(2 p+1) j}=\frac{L_{k,(2 p+1)(n+1)}+L_{k,(2 p+1) n}-2}{L_{k, 2 p+1}}+1
$$

In this case, the sum of the first k-Lucas numbers is (for $\mathrm{p}=0$ ),

$$
\sum_{j=0}^{n} L_{k, j}=\frac{1}{k}\left(L_{k, n+1}+L_{k, n}-2\right)+1
$$

that for the classical Lucas numbers is $\sum_{j=0}^{n} L_{j}=L_{n+2}-1$

\subsection{Corollary 2 (Sum of Consecutive Even k-Lucas Numbers)}

If $r=0$ and $a=2 p$, then Equation (2.2) is

$$
\sum_{j=0}^{n} L_{k, 2 p j}=\frac{L_{k, 2 p(n+1)}-L_{k, 2 p n}}{L_{2 p}-2}+1
$$

In this case, if $p=1$ we obtain the formula for the sum of the first even k-Lucas numbers $\sum_{j=0}^{n} L_{k, 2 j}=\frac{L_{k, 2 n+1}}{k}+1$, 
and for the classical Lucas numbers is $\sum_{j=0}^{n} L_{2 j}=L_{2 n+1}+1$

$$
\begin{aligned}
& \sum_{j=0}^{n}(-1)^{j} L_{k, a j+r} \\
& =\frac{(-1)^{a+n} L_{k, a n+r}+(-1)^{n} L_{k, a(n+1)+r}+(-1)^{n} L_{k, a-r}+L_{k, r}}{L_{k, a}+(-1)^{a}+1}
\end{aligned}
$$

\subsection{Theorem 3 (Sum of Alternated k-Lucas Numbers of Arithmetic Index)}

For $a>0$ and $r=0,1,2, \ldots a-1$, the sum of alternated $\mathrm{k}$-Lucas numbers is

Proof. As in the previous theorem,

$$
\begin{aligned}
& \sum_{j=0}^{n}(-1)^{j} L_{k, a j+r}=\sum_{j=0}^{n}(-1)^{j}\left(\sigma_{1}^{a j+r}+\sigma_{2}^{a j+r}\right) \\
& =\frac{(-1)^{n} \sigma_{1}^{a n+r}\left(-\sigma_{1}^{a}\right)-\sigma_{1}^{r}}{-\sigma_{1}^{a}-1}+\frac{(-1)^{n} \sigma_{2}^{a n+r}\left(-\sigma_{2}^{a}\right)-\sigma_{2}^{r}}{-\sigma_{2}^{a}-1} \\
& =\frac{(-1)^{n}(-1)^{a} \sigma_{1}^{a n+r}+(-1)^{n} \sigma_{1}^{a(n+1)+r}+(-1)^{r} \sigma_{2}^{a-r}+\sigma_{1}^{r}+(-1)^{n}(-1)^{a} \sigma_{2}^{a n+r}+(-1)^{n} \sigma_{2}^{a(n+1)+r}+(-1) \sigma_{1}^{a-r}+\sigma_{2}^{r}}{(-1)^{a}+\sigma_{1}^{a}+\sigma_{2}^{a}+1} \\
& =\frac{(-1)^{a}\left(\sigma_{1}^{a n+r}+\sigma_{2}^{a n+r}\right)+(-1)^{n}\left(\sigma_{1}^{a(n+1)+r}+\sigma_{2}^{a(n+1)+r}\right)+(-1)^{r}\left(\sigma_{2}^{a-r}+\sigma_{1}^{a-r}\right)+\sigma_{1}^{r}+\sigma_{2}^{r}}{L_{k, a}+(-1)^{a}+1} \\
& =\frac{(-1)^{a+n} L_{k, a n+r}+(-1)^{n} L_{k, a(n+1)+r}+(-1)^{n} L_{k, a-r}+L_{k, r}}{L_{k, a}+(-1)^{a}+1}
\end{aligned}
$$

\subsection{Corollary 3 (Sum of Consecutive Alternated Odd k-Lucas Numbers)}

As particular case, if $a=2 p+1$ and $r=0$,

$$
\begin{aligned}
& \sum_{j=0}^{n}(-1)^{j} L_{k,(2 p+1) j} \\
& =(-1)^{n} \frac{L_{k,(2 p+1)(n+1)}-L_{k,(2 p+1) n}+2(-1)^{n}}{L_{k, 2 p+1}}+1
\end{aligned}
$$

Then, for $p=0$ we obtain the sum of the first alternated $k$-Lucas numbers

$\sum_{j=0}^{n}(-1)^{j} L_{k, 2 j+1}=(-1)^{n} \frac{L_{k, n+1}-L_{k, n}+2(-1)^{n}}{k}+1$, that for

the classical Lucas numbers is

$$
\sum_{j=0}^{n}(-1)^{j} L_{2 j+1}=(-1)^{n} L_{k, n-1}+3 .
$$

$\sum_{j=0}^{n}(-1)^{j} L_{k, 2 j}=(-1)^{n} \frac{L_{k, 2(n+1)}+L_{k, 2 n}+2(-1)^{n}+k^{2}+2}{k^{2}+4}$,

that for the classical Lucas numbers is

$$
\sum_{j=0}^{n}(-1)^{j} L_{2 j}=(-1)^{n} \frac{1}{5}\left(5 F_{2 n+1}+3+2(-1)^{n}\right) .
$$

\section{On the k-Fibonacci Numbers of Indexes n and the k-Lucas Numbers}

In this section we will study a relation between the numbers $F_{k, 2^{r} n}$ and $L_{k, n}$.

\subsection{Theorem 4 (A Relation between Some k-Fibonacci and the k-Lucas Numbers)}

For $r \geq 1$, it is

$$
\frac{F_{k, 2^{r} n}}{F_{k, n}}=\prod_{j=0}^{r-1} L_{k, 2^{j} n}
$$

Proof.

$$
\begin{aligned}
& \frac{F_{k, 2^{r} n}}{F_{k, n}}=\frac{\sigma_{1}^{2^{r} n}-\sigma_{2}^{2^{r} n}}{\sigma_{1}^{n}-\sigma_{2}^{n}}=\left(\sigma_{1}^{2^{r-1} n}+\sigma_{2}^{2^{r-1} n}\right) \frac{\sigma_{1}^{2^{r-1} n}-\sigma_{2}^{2^{r-1} n}}{\sigma_{1}^{n}-\sigma_{2}^{n}} \\
& =L_{k, 2^{r-1} n}\left(\sigma_{1}^{2^{r-2} n}+\sigma_{2}^{2^{r-2} n}\right) \frac{\sigma_{1}^{2^{r-2} n}-\sigma_{2}^{2^{r-2} n}}{\sigma_{1}^{n}-\sigma_{2}^{n}} \\
& =L_{k, 2^{r-1} n} L_{k, 2^{r-2} n} \cdots \frac{\sigma_{1}^{2 n}-\sigma_{2}^{2 n}}{\sigma_{1}^{n}-\sigma_{2}^{n}}=\prod_{j=0}^{r-1} L_{k, 2^{j} n}
\end{aligned}
$$


In particular, if $r=1$, it is $\frac{F_{k, 2 n}}{F_{k, n}}=L_{k, n}$

Taking into account $L_{k, m}=\sigma_{1}^{m}+\sigma_{2}^{m}$, if we expand Formula (3.1), we find that this formula can be expressed as $\frac{F_{k, 2^{r} n}}{F_{k, n}}=\prod_{j=0}^{2^{r-1}-1}(-1)^{(j+1) n} L_{k, 2(2 j+1) n}$ or, that is the same, $\frac{F_{k, 2^{r} n}}{F_{k, n}}=\prod_{j=0}^{2^{r-2}-1} L_{k, 2(4 j+3) n}+(-1)^{n} \sum_{j=0}^{2^{r-1}-1} L_{k,(4 j+1) n}$

Then, applying Formula (2.2) to the second hand right of this equation with $n=2^{r-2}-1, a=4 n$, and $r=3 n$ for the first term and $r=n$ for the second,

$$
\begin{aligned}
& \frac{F_{k, 2^{r} n}}{F_{k, n}}=\frac{L_{k,\left(2^{r}+3\right) n}-L_{k,\left(2^{r}-1\right) n}+(-1)^{n} L_{k, n}-L_{k, 3 n}}{L_{k, 4 n}-2} \\
& +(-1)^{n} \frac{L_{k,\left(2^{r}+1\right) n}-L_{k,\left(2^{r}-3\right) n}+(-1)^{n} L_{k, 3 n}-L_{k, n}}{L_{k, 4 n}-2} \\
& =\frac{L_{k,\left(2^{r}+3\right) n}-L_{k,\left(2^{r}-1\right) n}+(-1)^{n}\left(L_{k,\left(2^{r}+1\right) n}-L_{k,\left(2^{r}-3\right) n}\right)}{L_{k, 4 n}-2}
\end{aligned}
$$

We tray to simplify the second hand right of this equation. For that, we will prove the following Lemma.

\subsection{Lemma 1}

$$
L_{k,(a+4) n}-L_{k, a n}=\left(k^{2}+4\right) F_{k,(a+2) n} F_{k, 2 n}
$$

Proof. We will apply the following formulas:

$$
\begin{aligned}
& L_{k, p}=F_{k, p-1}+F_{k, p+1} \quad \text { (relation) } \\
& F_{k,-p}=(-1)^{p+1} F_{k, p} \quad \text { (negative) } \\
& F_{k, a+b}=F_{k, a} F_{k, b-1}+F_{k, a+1} F_{k, b} \quad \text { (convolution) } \\
& F_{k, p+1}=k F_{k, p}+F_{k, p-1} \quad \text { (definition) }
\end{aligned}
$$

Then:

$$
\begin{aligned}
& L_{k,(a+4) n}-L_{k, a n} \\
& =F_{k,(a+4) n-1}+F_{k,(a+4) n+1}-F_{k, a n-1}-F_{k, a n+1} \\
& =F_{k,(a+2) n+(2 n-1)}+F_{k,(a+2) n+(2 n+1)} \\
& -F_{k,(a+2) n+(-2 n-1)}-F_{k,(a+2) n+(-2 n+1)} \\
& =F_{k,(a+2) n} F_{k, 2 n-2}+F_{k,(a+2) n+1} F_{k, 2 n-1} \\
& +F_{k,(a+2) n} F_{k, 2 n}+F_{k,(a+2) n+1} F_{k, 2 n+1} \\
& -F_{k,(a+2) n} F_{k,-2 n-2}-F_{k,(a+2) n+1} F_{k,-2 n-1} \\
& -F_{k,(a+2) n} F_{k,-2 n}-F_{k,(a+2) n+1} F_{k,-2 n+1}
\end{aligned}
$$

$$
\begin{aligned}
& =F_{k,(a+2) n} F_{k, 2 n-2}+F_{k,(a+2) n+1} F_{k, 2 n-1} \\
& +F_{k,(a+2) n} F_{k, 2 n}+F_{k,(a+2) n+1} F_{k, 2 n+1} \quad \text { (by negative) } \\
& +F_{k,(a+2) n} F_{k, 2 n+2}-F_{k,(a+2) n+1} F_{k, 2 n+1} \\
& +F_{k,(a+2) n} F_{k, 2 n}-F_{k,(a+2) n+1} F_{k, 2 n-1} \\
= & F_{k,(a+2) n}\left(F_{k, 2 n}-k F_{k, 2 n-1}+F_{k, 2 n}+k F_{k, 2 n+1}+F_{k, 2 n}\right) \\
= & F_{k,(a+2) n}\left(k^{2}+4\right) F_{k, 2 n}
\end{aligned}
$$

(by definition)

And applying this Lemma to Equation (3.2), we will have:

$$
\begin{aligned}
& \frac{F_{k, 2^{r} n}}{F_{k, n}} \\
& =\frac{\left(k^{2}+4\right) F_{k,\left(2^{r}+1\right) n} F_{k, 2 n}+(-1)^{n}\left(k^{2}+4\right) F_{k,\left(2^{r}-1\right) n} F_{k, 2 n}}{L_{k, 4 n}-2},
\end{aligned}
$$

that is

$$
\frac{F_{k, 2^{r} n}}{F_{k, n}}=\frac{\left(k^{2}+4\right) F_{k, 2 n}}{L_{k, 4 n}-2}\left(F_{k,\left(2^{r}+1\right) n}+(-1)^{n} F_{k,\left(2^{r}-1\right) n}\right)
$$

from where

$$
\begin{aligned}
& \frac{F_{k,\left(2^{r}+1\right) n}+(-1)^{n} F_{k,\left(2^{r}-1\right) n}}{F_{k, 2^{r} n}}=\frac{L_{k, 4 n}-2}{\left(k^{2}+4\right) F_{k, n} F_{k, 2 n}} \\
& =\frac{L_{k, 4 n}-2}{\left(\sigma_{1}^{n}-\sigma_{2}^{n}\right)\left(\sigma_{1}^{2 n}-\sigma_{2}^{2 n}\right)} \\
& =\frac{L_{k, 4 n}-2}{\left(\sigma_{1}^{3 n}+\sigma_{2}^{3 n}\right)-(-1)^{n}\left(\sigma_{1}^{n}+\sigma_{2}^{n}\right)}=\frac{L_{k, 4 n}-2}{L_{k, 3 n}-(-1)^{n} L_{k, n}}
\end{aligned}
$$

If in Equation (3.3) it is a $=0$, then it is $F_{k, 2 n}^{2}=\frac{L_{k, 4 n}-2}{k^{2}+4}$, and applying the Formulas (2.5) and (2.4),

$$
\begin{aligned}
\sum_{j=0}^{n} F_{k, 2 j}^{2} & =\frac{1}{k^{2}+4} \sum_{j=0}^{n}\left(L_{k, 4 j}-2\right) \\
& =\frac{1}{k^{2}+4}\left(\frac{L_{k, 4(n+1)}-L_{k, 4 n}}{L_{k, 4}-2}+1-2(n+1)\right)
\end{aligned}
$$

That is

$$
\sum_{j=0}^{n} F_{k, 2 j}^{2}=\frac{1}{k^{2}+4}\left(\frac{k L_{k, 4 n+3}+L_{k, 4 n+1}}{k\left(k^{2}+4\right)}-2 n-1\right)
$$

In particular, for the classical Lucas numbers $(\mathrm{k}=1)$, it is $\sum_{j=0}^{n} F_{2 j}^{2}=\frac{1}{5}\left(\frac{L_{4 n+3}+L_{4 n+1}}{5}-2 n-1\right)$. 


\section{Acknowledgements}

This work has been supported in part by CICYT Project number MTM200805866-C03-02 from Ministerio de Educación y Ciencia of Spain.

\section{REFERENCES}

[1] S. Falcon, "On the k-Lucas Numbers," International Journal of Contemporary Mathematical Sciences, Vol. 6, No. 21, 2011, pp. 1039-1050
[2] S. Falcon and A. Plaza, "On the Fibonacci k-Numbers," Chaos, Solitons \& Fractals, Vol. 32, No. 5, 2007, pp. 1615-1624. doi:10.1016/j.chaos.2006.09.022

[3] S. Falcon and A. Plaza, "The k-Fibonacci Sequence and the Pascal 2-Triangle," Chaos, Solitons \& Fractals, Vol. 33 , No. 1,2007 , pp. 38-49. doi:10.1016/j.chaos.2006.10.022

[4] S. Falcon and A. Plaza, "On k-Fibonacci Numbers of Arithmetic Indexes," Applied Mathematics and Computation, Vol. 208, 2009, pp. 180-185 doi:10.1016/j.amc.2008.11.031 